\title{
A Tunable Energy Filter for Medical X-Ray Imaging
}

\author{
Erik Fredenberg, ${ }^{1}$ Björn Cederström, ${ }^{1}$ Magnus Åslund, ${ }^{2}$ \\ Carolina Ribbing, ${ }^{3}$ and Mats Danielsson ${ }^{1}$ \\ ${ }^{1}$ Department of Physics, Royal Institute of Technology, AlbaNova University Center, 10691 Stockholm, Sweden \\ ${ }^{2}$ Research \& Development Department, Sectra Mamea AB, Smidesvägen 5, 17141 Solna, Sweden \\ ${ }^{3}$ Department of Engineering Sciences, The Angström Laboratory, Uppsala University, 75121 Uppsala, Sweden
}

Correspondence should be addressed to Erik Fredenberg, fberg@mi.physics.kth.se

Received 18 April 2008; Accepted 14 August 2008

Recommended by Doug Pfeiffer

A multiprism lens (MPL) is a refractive X-ray lens, and its chromatic properties can be employed in an energy filtering setup to obtain a narrow tunable X-ray spectrum. We present the first evaluation of such a filter for medical X-ray imaging. The experimental setup yields a 6.6 gain of flux at $20 \mathrm{keV}$, and we demonstrate tunability by altering the energy spectrum to center also around 17 and $23 \mathrm{keV}$. All measurements are found to agree well with ray-tracing and a proposed geometrical model. Compared to a model mammography system with absorption filtering, the experimental MPL filter reduces dose $13-25 \%$ for 3-7 cm breasts if the spectrum is centered around the optimal energy. Additionally, the resolution is improved 2.5 times for a $5 \mathrm{~cm}$ breast. The scan time is increased 3 times but can be reduced with a slightly decreased energy filtering and resolution.

Copyright () 2008 Erik Fredenberg et al. This is an open access article distributed under the Creative Commons Attribution License, which permits unrestricted use, distribution, and reproduction in any medium, provided the original work is properly cited.

\section{Introduction}

The absorbed dose necessary to obtain a medical X-ray image with sufficient signal-to-noise ratio varies with object composition, thickness, and X-ray energy spectrum [1-3]. In fact, for a certain object composition and thickness there exists an optimal energy; low-energy photons are to a great extent absorbed and contribute to dose, whereas photons of higher energies pass the object unaffected or scattered and add to noise without giving much useful information. A spectrum that is narrow and centered around the optimal energy is hence the most dose-efficient one.

The positive effects of energy filtering to optimize the spectrum were observed already at the advent of medical Xray imaging. It was noted that putting a thin layer of material, such as a piece of leather, between the patient and the X-ray tube resulted in less irritation of the patient's skin [4]. Since then, energy filtering has not changed drastically. Absorption filtering is still the dominant method to filter out low-energy photons, often in combination with a limited X-ray tube acceleration voltage to cut off photons of higher energy. In some cases the material of the absorption filter is chosen so as to have an absorption edge above the optimal energy to further reduce the high-energy part of the spectrum. In principle, the spectrum can be made arbitrarily narrow using heavy filtration, but only at the cost of a severe reduction in flux.

To optimize the spectrum beyond what is practically achievable with absorption filtering, several options have been proposed for mammography and computed tomography. Some X-ray sources are capable of producing a high enough flux to allow for very efficient monochromators, for example, synchrotrons [5-7], laser plasma sources [8], channeling radiation sources [9], parametric X-ray sources [10], and sources producing X-rays by inverse Compton scattering on free electron lasers [11]. Authors have reported promising results as to image quality and dose efficiency. Nevertheless, the high complexity and cost of such sources limit the feasibility for routine clinical X-ray imaging.

It is also possible to apply less stringent energy filtering to a regular X-ray tube, and filtering based on mosaic crystals has, for instance, been proposed for mammography [12, 13]. Such crystals have small imperfections in the structure, resulting in a broader energy spectrum and higher flux 
compared to perfect crystals. The reduced flux compared to conventional mammography is, however, still identified as a problem, and a decreased resolution in the plane of diffraction of the crystal has been noted. To somewhat mitigate the reduction in flux, mosaic crystals can be used in combination with polycapillary optics to gather the radiation into a quasiparallel beam [14].

The multiprism lens (MPL) is a refractive X-ray lens. It distinguishes itself from other X-ray lenses, such as parabolic lenses, compound refractive lenses, and fresnel lenses, by a simplified manufacturing process, and a tunable focal length [15]. Experimental measurements have been performed at synchrotron facilities for various lens materials such as epoxy and silicon [16], and lithium [17]. Studies have also been performed with X-ray tubes using MPLs made of, for example, vinyl [15] and plexiglas [18].

Chromatic properties of the MPL make it possible to employ it as an X-ray energy filter, and in the following, we present a study of such an MPL filter. It is intended as an alternative to the mentioned filtering methods for small-scale applications, such as medical imaging, and we use an X-ray tube for the evaluation. We construct theoretical models of the MPL filter and compare the results to measurements. We show tunability in a fixed geometry for the first time experimentally, and we estimate the feasibility and potential advantages of introducing MPL filters in medical imaging.

\section{Methods}

\subsection{Background}

MPLs consist of two rows of prisms put on an angle in relation to each other and symmetrically arranged around the optical axis. The rows touch at the entrance side of the lens and are separated from the optical axis by a distance $d_{g}$ at the exit side (Figure 1). Peripheral rays entering the lens encounter a larger number of prisms than central ones and are therefore refracted more. It can be shown that the amount of lens material in the direction of the optical axis has a parabolic projection [16]. Since MPLs consist of only flat surfaces, manufacturing is relatively easy, and the lenses are therefore suitable for small-scale applications.

The focal length of an MPL with length $L$ and tooth height $d_{t}$ is $F=d_{g} d_{t} / \delta L$, where $\delta$ is the decrement of the real part of the refractive index from unity [16]. Imaging is onedimensional and follows the Gaussian lens formula for a thin lens, $F^{-1}=s_{o}^{-1}+s_{i}^{-1}$, where $s_{o}$ and $s_{i}$ are the object and image distances, respectively. $F$ can be made short (in the order of $0.1 \mathrm{~m}$ ), but note that the parabolic projection holds over the whole aperture only as long as $d_{g}$ is larger than $d_{t}$.

Since $\delta$ in the considered energy interval varies as $E^{-2}$, the lens is chromatic. Thus, if a slit is placed in the image plane of a particular X-ray energy $\left(E_{\text {peak }}\right)$, radiation of that energy is transmitted through the slit, whereas other energies are out of focus and preferentially blocked (Figure 1). The focal length, and so $E_{\text {peak }}$, can be tuned by changing $d_{g}$ in an otherwise fixed geometry. Henceforth, the MPL and slit together are referred to as the MPL filter.

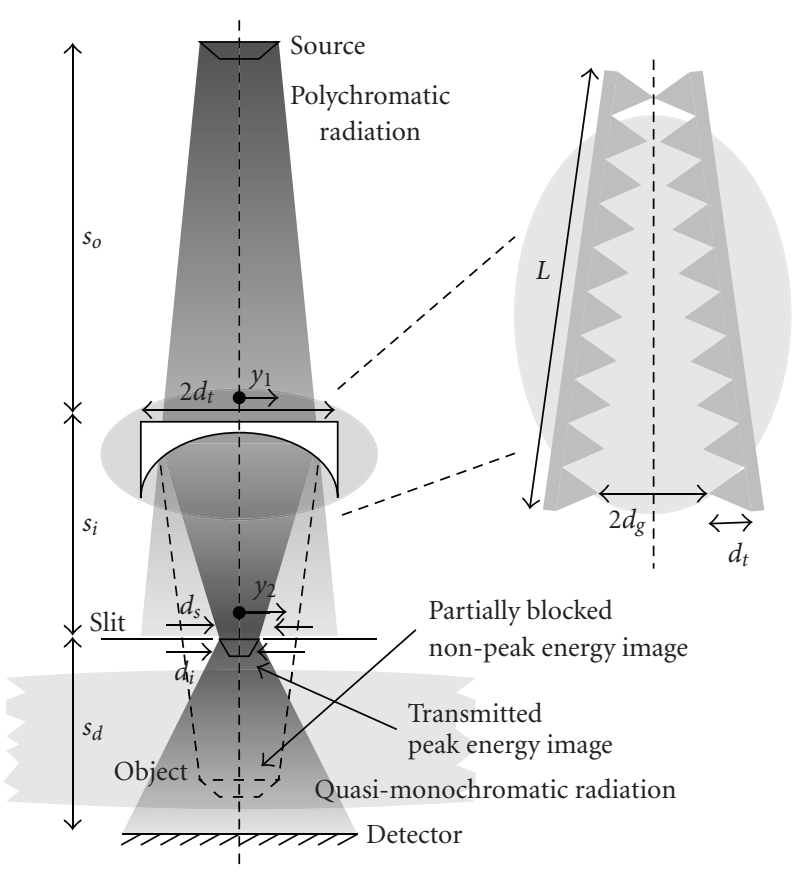

FIGURE 1: Left: energy filtering with the multiprism lens. The chromatic lens focuses radiation on a slit, and the energy corresponding to a sharp image is preferentially transmitted. We use a setup with $s_{o}=755 \mathrm{~mm}$ and $s_{i}=314 \mathrm{~mm}$, yielding an image size of $d_{i}=d_{s}=$ $10.0 \mu \mathrm{m}$ with a $24 \mu \mathrm{m}$ source. Right: close-up of the lens. It has a tooth height $d_{t}=100 \mu \mathrm{m}$ a length $L=61 \mathrm{~mm}$, and a focal length that is tuned by changing $d_{g}$.

A primary figure of merit of the MPL filter is the energy dependent gain of flux, which is the transmitted flux from radiation focused by the MPL $\left(\Phi_{\text {foc }}\right)$ normalized to the flux from divergent radiation in an identical setup but without lens $\left(\Phi_{\text {div }}\right)$;

$$
G(E)=\frac{\Phi_{\mathrm{foc}}(E)}{\Phi_{\mathrm{div}}(E)} .
$$

An important MPL parameter is the effective aperture $\left(D_{e}\right)$, which is the width of a slit with the same transmission as the lens. Dividing the effective aperture by the physical aperture of the lens yields the average transmission $\left(N_{t}\right)$. Both these parameters quantify absorption in the MPL.

In this study, we chose to investigate an epoxy MPL $(Z \approx 6)$. Beryllium or lithium with lower atomic numbers might yield better results, but because of manufacturing and handling problems, these lens materials are ruled out for small-scale applications.

\subsection{Geometrical and Ray-Tracing Models}

We derive a theoretical model of $G(E), D_{e}$, and $N_{t}$, based on geometrical optics and a thin lens approximation. In an energy filtering setup according to Figure 1 with a lens of tooth height $d_{t}$, the transmitted flux can be described as

$$
\Phi_{\text {foc }}(E)=\Phi_{0}(E) \frac{1}{2 \pi} \int_{-d_{t}}^{d_{t}} \frac{\eta_{t}(E) \eta_{i}(E)}{s_{o}} \mathrm{~d} y_{1},
$$


where $\eta_{t}$ and $\eta_{i}$ account for transmission through the lens and the slit, respectively, $\Phi_{0}$ is the X-ray tube flux, $y_{1}$ refers to the lateral position in the plane of the lens, and the integral is taken over the lens aperture.

An MPL with linear attenuation coefficient $\mu$ has a Gaussian transmission profile

$$
\eta_{t}(E)=\exp \left(-\frac{y_{1}^{2}}{2 \sigma^{2}(E)}\right)
$$

with $\operatorname{rms} \sigma=\sqrt{F \delta / \mu}[16]$.

If the image of the source from each point $y_{1}$ on the lens is described by the function $f_{i}\left(E, y_{1}, y_{2}\right)$, the part of the radiation that is not blocked by the slit is $f_{i}$ integrated over the slit opening $\left(d_{s}\right)$,

$$
\eta_{i}(E)=\int_{d_{s}} f_{i}\left(y_{1}, y_{2}, E\right) \mathrm{d} y_{2},
$$

where $y_{2}$ refers to the lateral position in the plane of the slit. In this plane, which is the image plane of $E_{\text {peak }}, f_{i}\left(E_{\text {peak }}, y_{1}, y_{2}\right)$ from all points on the lens coincide to form a sharp image, whereas $f_{i}$ contributions from any other energy are spread out and sum to a blurred total image.

The flux from divergent radiation through the slit can be calculated as

$$
\Phi_{\mathrm{div}}(E)=\Phi_{0}(E) \frac{1}{2 \pi} \frac{d_{s}}{s_{o}+s_{i}}
$$

by assuming a point source.

Equations (2) and (5) yield the gain according to (1). The effective aperture is obtained by integrating (3) over the physical lens aperture, which in turn yields the average transmission;

$$
N_{t}(E)=\frac{D_{e}(E)}{2 d_{t}}, \quad \text { where } D_{e}(E)=\int_{-d_{t}}^{d_{t}} \eta_{t}(E) \mathrm{d} y_{1} .
$$

The described geometrical model assumes the lens to be thin and free of any aberrations except its chromaticity. To estimate the impact of those approximations, we implement the setup in a custom-made ray-tracing model using the MATLAB software package (The MathWorks Inc., Natick, Mass, USA). A large number of rays $\left(\sim 10^{6}\right)$ emerging from the source at random positions and angles in energy steps of $0.5 \mathrm{keV}$ are traced through each tooth of the lens. Published linear absorption coefficients [19] and semiempirical data on atomic scattering factors [20] serve as input to calculate absorption and refraction. Compton scattering is treated as absorption, and the lens is assumed flawless.

\subsection{Measurements}

The experimental setup is depicted in Figure 2. We use a tungsten anode X-ray tube (Philips PW2274/20), which is water cooled and can be run continuously. The acceleration voltage for all measurements is $33 \mathrm{kVp}$, the anode current is $10 \mathrm{~mA}$, and the anode angle is $3.5^{\circ}$. At this angle, the X-ray tube focal spot size is $24 \mu \mathrm{m}$, as specified by the manufacturer.

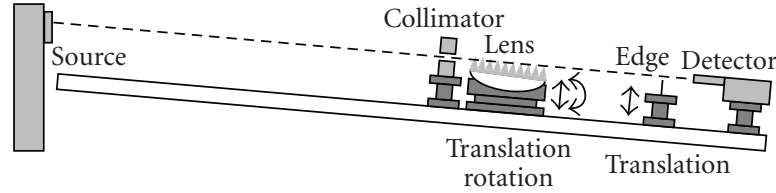

FIgURE 2: Schematic of the experimental setup used for the measurements. To simplify alignment, we measure on only one lens-half, and instead of using a slit, a tantalum edge is scanned across the image plane.

An edge scan of the source confirmed the size to be in the interval $22-27 \mu \mathrm{m}$ FWHM. The shape is approximately trapezoidal with a base-to-FWHM ratio of 1.6.

The epoxy MPL was molded from a silicon master, which in turn was produced by wet anisotropic etching [16]. It has a tooth height of $d_{t}=100 \mu \mathrm{m}$ and a length of $L=61 \mathrm{~mm}$. To simplify alignment, we use only one lens-half, which does not change anything in principle but reduces the gain by a factor of two. The lens-half is mounted on precision stages for lateral translation and tilting, and it is collimated by a $100 \mu \mathrm{m}$ collimator slit.

We use a CZT compound solid-state detector (Amptek XR-100T-CZT) to record the energy spectrum transmitted through the filter. It has a near $100 \%$ detection efficiency, negligible hole-tailing, and an energy resolution better than $0.5 \mathrm{keV}$ around $20 \mathrm{keV}$.

The distances $s_{0}=755 \mathrm{~mm}$ and $s_{i}=314 \mathrm{~mm}$ are chosen in accordance with the Gaussian lens formula so as to produce a $d_{i}=10.0 \mu \mathrm{m}$ image of the source. We obtain the image of the source by scanning a tantalum edge in steps of $1 \mu \mathrm{m}$ over the image plane and differentiating the recorded profile.

To find $\Phi_{\text {foc }}$, we fit a straight line to the edge scan profile over the interval with the fastest rate of change. The slope of the line is the derivative over an interval where the scan profile is essentially linear, and the length of the line corresponds to the size of the slit. To determine $\Phi_{\text {div }}$, the same procedure is repeated without an MPL. The gain is calculated from $\Phi_{\text {foc }}$ and $\Phi_{\text {div }}$, and $E_{\text {peak }}$ is at the maximum gain. We do not use a slit in the image plane of our setup to avoid the additional uncertainty introduced by a narrow slit. $N_{t}$ is measured without the edge as the fraction of flux with and without lens. $D_{e}$ is calculated from $N_{t}$.

We demonstrate tunability of the MPL filter by repeating the measurements for several angles, corresponding to a range of peak energies. The absolute gap at the rear end of the lens $\left(d_{g}\right)$ cannot be determined by the experiment, but the relative gap between the measurements $\left(\Delta d_{g}\right)$ is monitored.

\subsection{Medical Imaging}

To evaluate the MPL filter for medical imaging, we use a theoretical model of a scanning digital mammography system and compare an array of filters (the MPL system) to the same number of precollimators in an absorption-filtered multislit geometry (the reference system). The comparison is made in terms of dose efficiency, scan time, and resolution. 




○ Measurement

Fit

-- \pm 1 standard deviation

FIgUre 3: An image of the source at $E_{\text {peak }}=20 \mathrm{keV}$. The differentiated edge-scan profile is fitted to a $11.0 \mu \mathrm{m}$ FWHM trapezoid. The dotted profiles correspond to \pm 1 standard deviation of the fitted width.

A scanning system is ideal for implementation of the MPL filter since the line focus of each lens can be matched to a linear detector. One example of a clinical mammography system based on a scanning multislit geometry is the Sectra MicroDose Mammography (MDM) system (Sectra Mamea AB, Kista, Sweden) [21-23].

The reference system, similar but not identical to the MDM system, is absorption filtered and has a $450 \times 600 \mu \mathrm{m}^{2}$ $\mathrm{X}$-ray tube spot size. Each detector has a corresponding $60 \mu \mathrm{m}$ precollimator slit, which is placed before the object, $650 \mathrm{~mm}$ from the source, and $100 \mathrm{~mm}$ upstream of the detector. The point spread function (PSF) of the system is calculated as a convolution of the slit and the source, using the measured source shape.

In the MPL system, each lens-detector pair is similar to the setup in Figure 1, with a distance $s_{d}=100 \mathrm{~mm}$ for the object between the slit and the detector. The PSF is the image of the source when assuming perfect lens imaging.

The modulation transfer functions (MTFs) of the systems are calculated as the Fourier transforms of the respective PSFs, and we define the resolution in the scanning direction $(v)$ as the spatial frequency in the detector plane when the MTF has dropped to 0.1 . In the direction perpendicular to the scanning (parallel to the lenses or slits), the width of the source in the MPL system is chosen so that the resolution of the two systems is equal. A published method to find the maximum load to rotary tungsten anodes as a function of source size [24] is used to calculate the relative scan time ( $\left.\widehat{T}=T_{\mathrm{mpl}} / T_{\text {ref }}\right)$.

In both systems, the object used for the calculations consists of breast tissue of $50 \%$ glandularity with a $5 \mathrm{~mm}$ skin layer and an embedded $300 \mu \mathrm{m}$ microcalcification. The image receptor is a $3.6 \mathrm{~mm}$ thick silicon-strip detector, which affects the quantum efficiency but is assumed ideal in terms of resolution.

To estimate dose efficiency, we use the spectral quantum efficiency (SQE) as a figure of merit. It is defined as [22]

$$
\mathrm{SQE}=\frac{\mathrm{SDNR}^{2}}{\mathrm{AGD}} \cdot \frac{\mathrm{AGD}_{\text {mono }}}{\mathrm{SDNR}_{\text {mono }}^{2}},
$$

calculated with the signal-difference-to-noise ratio (SDNR) between the microcalcification and surrounding tissue, and the average glandular dose (AGD). The SQE is normalized to the ideal monochromatic case. For a quantum limited system and a given spectrum, it is an exposure-independent quantity, inversely proportional to the dose needed to obtain a certain SDNR.

Normalized glandular dose coefficients [25] and Xray attenuation coefficients [19] are used to calculate dose and absorption. For anode materials other than tungsten, published spectra are used as input [26]. The influence of scattering is neglected, which is a fair assumption for a multislit geometry [23].

\section{Results}

\subsection{Filter Parameters}

Measurements are presented at $E_{\text {peak }}=23,20$, and $17 \mathrm{keV}$. Table 1 shows the corresponding values of $d_{g}$ for the models, and the measured $\Delta d_{g}$ to obtain the 20 and $17 \mathrm{keV}$ peaks relative to the $23 \mathrm{keV}$ peak. $\Delta d_{g}$ agrees within $1 \mu \mathrm{m}$ with the geometrical model, and within $2 \mu \mathrm{m}$ with ray-tracing.

Figure 3 shows the sharp image of the source at $20 \mathrm{keV}$ as determined by the differentiated edge-scan, fitted to a trapezoid with a similar shape as the source. The FWHMs of the fitted profiles $\left(d_{i}\right)$ for all three measurements are tabulated in Table 1 and compared to model predictions. A somewhat smaller $d_{i}$ is expected from the models, but the discrepancy is not large and falls within one standard deviation of the fit. Small-angle scattering, which is treated as absorption in the models, and air bubbles in the epoxy might account for parts of the increased image size.

As seen in Table $1, N_{t}$ in the considered interval is almost independent of lens angle with a measured mean of 0.52. $D_{e}$ is thus slightly more than half the physical aperture.

The transmitted flux through a $10 \mu \mathrm{m}$ slit as a function of energy is plotted in Figure 4, with an MPL $\left(\Phi_{\text {foc }}\right)$ and without any filter $\left(\Phi_{\text {div }}\right)$. Also shown is $\Phi_{\text {div }}$ normalized to the peak of $\Phi_{\text {foc }}$ to illustrate the difference in shape. $\Phi_{\text {foc }}$ and $\Phi_{\text {div }}$ yield the gain by division, with the result in Figure 5 for $E_{\text {peak }}=$ 23,20 , and $17 \mathrm{keV}$ along with the predicted results from the models. Also shown in the figure is the calculated gain for 22 and $27 \mu \mathrm{m}$ sources, which corresponds to one standard deviation of the source size measurements. It is evident that the gain decreases with the source size, and the uncertainty in the source size measurement therefore adds an additional overall uncertainty to the models.

Values of the peak gain and deviations of the measured values from the models are presented in Table 1. Since the ray-tracing results agree well with the measurements, 




Figure 4: The measured MPL-filtered spectrum at $E_{\text {peak }}=20 \mathrm{keV}$ $\left(\Phi_{\mathrm{foc}}\right)$ compared to the unfiltered $33 \mathrm{kVp}$ tungsten spectrum $\left(\Phi_{\text {div }}\right)$. Also shown is $\Phi_{\text {div }}$ normalized to the peak value of $\Phi_{\text {foc }}$.

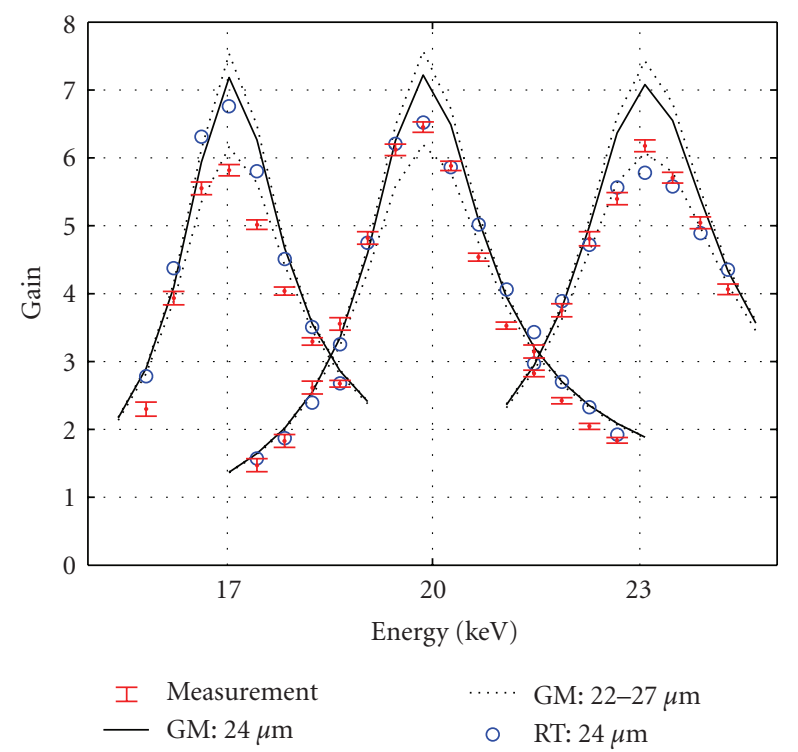

FIGURE 5: Gain of flux as a function of energy for $E_{\text {peak }}=17,20$, and $23 \mathrm{keV}$. The error bars correspond to \pm 1 standard deviation of the measured values. Also shown are results from the geometrical (GM) and ray-tracing (RT) models for a $24 \mu \mathrm{m}$ source, and the geometrical model for source sizes $22-27 \mu \mathrm{m}$.

deviations of the geometrical model can be explained to a large extent by model approximations.

\subsection{Medical Imaging}

Figure 6 shows the SQE as a function of breast thickness for the measured MPL-filtered spectra $\left(\Phi_{\text {foc }}\right)$ at $E_{\text {peak }}=17,20$, and $23 \mathrm{keV}$, and for a spectrum at $E_{\text {peak }}=25 \mathrm{keV}$ calculated

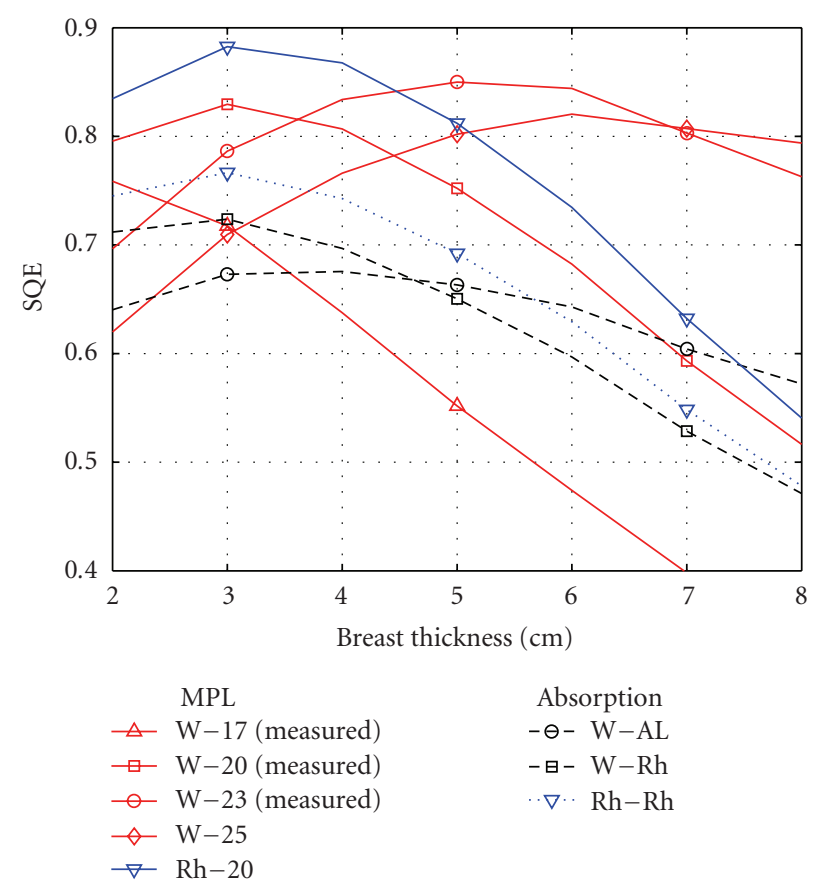

FIGURE 6: Filter performance in mammography using the SQE as a figure of merit. The measured and calculated MPL-filtered spectra are denoted by anode material $-E_{\text {peak }}$. Absorption filtered spectra are denoted by anode-filter materials. The aluminum and rhodium filters are $0.5 \mathrm{~mm}$ and $25 \mu \mathrm{m}$ thick.

with the geometrical model. The 20, 23, and $25 \mathrm{keV}$ spectra are optimized for breast thicknesses 3,5 , and $7 \mathrm{~cm}$ with SQE values of $0.83,0.85$, and 0.82 , respectively, whereas the $17 \mathrm{keV}$ spectrum falls outside the mammographic region. Figure 6 thus illustrates the importance of a tunable filter, in particular for thinner breasts.

Also plotted in Figure 6 is the SQE of the reference system with $0.5 \mathrm{~mm}$ aluminum and $25 \mu \mathrm{m}$ rhodium absorption filters, calculated with the measured tungsten spectrum $\left(\Phi_{\text {div }}\right)$ as input. The rhodium-filtered spectrum is optimal for a $3 \mathrm{~cm}$ breast, whereas the aluminum-filtered spectrum is relatively constant over the range of breast thicknesses. SQE values of $0.72,0.66$, and 0.61 are obtained with the best absorption filter for breast thicknesses of 3,5 , and $7 \mathrm{~cm}$. The MPL filter thus improves the SQE 15, 29, and 34\%, corresponding to dose reductions of 13,22 , and $26 \%$.

The SQE of the reference system can be raised by switching to a rhodium anode. That is, however, the case also for the MPL system and the improvement is still $15 \%$ for a $3 \mathrm{~cm}$ breast, hence independent of the anode material. A molybdenum-molybdenum anode-filter combination yields an SQE that is inferior or equal to the rhodium-rhodium spectrum across the range of breast thicknesses, and it is therefore not included in the comparison.

Table 2 shows $\nu, S Q E$, and $\widehat{T}$ for a $5 \mathrm{~cm}$ breast and a range of MPL-system parameters at $E_{\text {peak }}=23 \mathrm{keV}$. We used the measured MPL-filtered spectrum and the geometrical model to calculate $\hat{T}$ for the experimental and the hypothetical setups, respectively. The reference system employs 
TABLE 1: Summary of the measurements and model predictions. The average transmission $\left(N_{t}\right)$, image size $\left(d_{i}\right)$, gain $(G)$, and the gap at the rear end of the lens $\left(d_{g}\right)$ are presented at $E_{\text {peak }}=17,20$, and $23 \mathrm{keV}$.

\begin{tabular}{|c|c|c|c|c|c|c|c|c|c|c|c|c|c|c|}
\hline \multirow[b]{2}{*}{$\begin{array}{c}E_{\text {peak }} \\
{[\mathrm{keV}]}\end{array}$} & \multicolumn{4}{|c|}{ Geometrical model } & \multicolumn{4}{|c|}{ Ray tracing } & \multicolumn{6}{|c|}{ Measurement } \\
\hline & $\begin{array}{c}d_{i} \\
{[\mu \mathrm{m}]}\end{array}$ & $N_{t}$ & $\begin{array}{c}d_{g} \\
{[\mu \mathrm{m}]}\end{array}$ & $G_{\mathrm{GM}}$ & $\begin{array}{c}d_{i} \\
{[\mu \mathrm{m}]}\end{array}$ & $N_{t}$ & $\begin{array}{c}d_{g} \\
{[\mu \mathrm{m}]}\end{array}$ & $G_{\mathrm{RT}}$ & $\begin{array}{l}d_{i}^{(\mathrm{a})} \\
{[\mu \mathrm{m}]}\end{array}$ & $N_{t}$ & $\begin{array}{c}\Delta d_{g} \\
{[\mu \mathrm{m}]}\end{array}$ & $G_{M}^{(a)}$ & $\begin{array}{c}\frac{\Delta G}{G_{\mathrm{GM}}} \\
{[\%]}\end{array}$ & $\begin{array}{l}\frac{\Delta G}{G_{\mathrm{RT}}} \\
{[\%]}\end{array}$ \\
\hline 23 & 10.0 & 0.59 & 63 & 7.4 & 9.3 & 0.57 & 53 & 6.0 & $11.1(7.8-13.8)$ & 0.50 & 0 & $6.2 \pm 0.1$ & -16 & +3 \\
\hline 20 & 10.0 & 0.60 & 83 & 7.6 & 9.2 & 0.57 & 74 & 6.8 & $11.0(7.9-13.8)$ & 0.52 & 20 & $6.6 \pm 0.1$ & -13 & -2 \\
\hline 17 & 10.0 & 0.59 & 115 & 7.5 & 9.8 & 0.57 & 103 & 7.1 & $12.9(9.6-16.1)$ & 0.54 & 52 & $5.9 \pm 0.1$ & -21 & -17 \\
\hline
\end{tabular}

(a) Error estimates correspond to \pm 1 standard deviation.

a $0.5 \mathrm{~mm}$-aluminum-filtered tungsten spectrum. Since $v$ is in the scanning direction, it can be improved by oversampling.

Referring to Table 2, an MPL system with parameters corresponding to the current experimental setup yields a longer scan time than the reference system (row 1), but the resolution in the scanning direction is 2.5 times higher. By either increasing the size of the source (row 2) or by moving the lens closer to it (row 3), a scan time that matches the reference system is achieved. Compared to the reference system, the resolution is still 1.75-1.88 times higher. The SQE is $17-20 \%$ higher with corresponding dose reductions of 14 $16 \%$. Standard mammography systems are shorter than the setup we used for the experiment, but with a shorter focal length, a more compact MPL system is obtained (row 4). If the resolution of the two systems are matched (row 5), the MPL filter is able to reduce scan time compared to the reference system, still at an improved SQE.

\section{Discussion}

\subsection{Filter Parameters}

All aspects of the experimental results-image size, transmission, tunability, and gain - agree well with model predictions.

A possible reason for the relatively large deviation of the $17 \mathrm{keV}$ peak in terms of image size and gain is a sudden increase in size or a movement of the X-ray tube focal spot. The focal spot is affected by the cooling water temperature, which varied randomly during the several-hour measurements.

In an MPL, deflection occurs not only at two surfaces as in a parabolic lens, but all along the way through the lens. Central rays are refracted closer to the entrance side than peripheral rays, and the rear principal plane therefore depends on the lateral position of the incident ray. In addition, deflected rays miss teeth towards the exit side of the lens, and the projection for parallel incident rays is hence not entirely parabolic. Both these effects result in the same kind of aberration: an elongated focal length, in particular for peripheral rays, and, as a consequence, a stretched image in the direction of the optical axis.

The $\sim 10 \mu \mathrm{m}$ smaller $d_{g}$ predicted by ray-tracing compared to the geometrical model for a certain focal length (Table 1) can be explained by the fact that the former takes the elongated focal length into account, whereas the latter assumes the lens to be thin. It is likely that the measurements follow the ray-tracing model, although $d_{g}$ was not monitored directly. A smaller $d_{g}$ results in more absorption, which explains the somewhat lower transmission and gain from ray tracing and measurements compared to the geometrical model.

\subsection{Medical Imaging}

Table 2 shows that the MPL filter can reduce dose at the same scan time as the reference system, and if a longer scan time is accepted, the energy filtering in terms of SQE can be improved.

Note that the optimal energy is system specific; we might arrive at different results with detector materials other than silicon. Previous studies on spectrum optimization indicate that the optimal energy also depends on breast size and composition but is fairly independent of lesion type [3]. Our results for microcalcifications should therefore be similar for masses.

The presented values on the SQE for absorption-filtered spectra are slightly higher than previously reported results from a similar system [22]. The reason for the better dose efficiency is substantial self-filtration at the steep anode angle, and a correspondingly harder input spectrum. In addition, since the acceleration voltage is not tuned with respect to breast thickness in the present study, the SQE is less constant across the range of thicknesses. For an MPL system, tuning of the acceleration voltage is of less importance since the high-energy end of the spectrum is also filtered. One possibility is therefore to use a higher voltage with the MPL system to achieve higher flux at a constant or improved dose efficiency. This option is not further investigated here.

Other types of X-ray optics have been investigated for energy filtering in medical imaging, in particular mosaic crystals. The measured average transmission of the MPL is 0.54 at $17 \mathrm{keV}$, which for an MPL with two lens-halves corresponds to an effective aperture of $108 \mu \mathrm{m}$. Mosaic HOPG (002) crystals with a mosaic spread of $0.4^{\circ}$ give a similar energy spectrum as the MPL filter but a lower peak reflectivity at $17.5 \mathrm{keV}: 0.38$ according to theoretical estimates for an infinitely thick crystal [27], and 0.22 as measured in a set-up relevant for mammography [13]. At higher energies, the reflectivity drops further. The crystal can be expected to have a larger aperture than the MPL, 
TABLE 2: Comparison of a scanning MPL-filtered mammography set-up at $E_{\text {peak }}=23 \mathrm{keV}$ to a clinical reference system with absorption filtration for a $5 \mathrm{~cm}$ breast. The spectral quantum efficiency (SQE) and the relative scan time $(\widehat{T})$ are presented for the experimentally obtained spectrum (first row), along with a number of calculated MPL-filtered spectra (subsequent rows) with different focal lengths ( $F$ ), source-to-lens and lens-to-slit distances $\left(s_{o}\right.$ and $\left.s_{i}\right)$, and resolution in the scanning direction $(\nu)$. The parameters of the reference system are shown in the bottom row.

\begin{tabular}{|c|c|c|c|c|c|c|}
\hline $\begin{array}{c}F \\
{[\mathrm{~mm}]}\end{array}$ & $\begin{array}{c}\text { Source size } \\
{\left[\mu \mathrm{m}^{2}\right]}\end{array}$ & $\begin{array}{c}s_{o} \\
{[\mathrm{~mm}]}\end{array}$ & $\begin{array}{c}s_{i} \\
{[\mathrm{~mm}]}\end{array}$ & $\begin{array}{c}v \\
{\left[\mathrm{~mm}^{-1}\right]}\end{array}$ & SQE & $\widehat{T}$ \\
\hline 219 & $24 \times 932$ & 755 & 308 & 20 & 0.85 & 3.0 \\
\hline 219 & $65 \times 932$ & 755 & 308 & 14 & 0.77 & 1.0 \\
\hline 219 & $24 \times 812$ & 360 & 560 & 15 & 0.79 & 1.0 \\
\hline 153 & $24 \times 600$ & 245 & 405 & 12 & 0.77 & 1.0 \\
\hline 153 & $45 \times 600$ & 245 & 405 & 8 & 0.72 & 0.63 \\
\hline & $450 \times 600$ & \multicolumn{2}{|c|}{$650^{(\mathrm{a})}$} & 8 & 0.66 & 1.0 \\
\hline
\end{tabular}

(a) The source-to-collimator distance.

but that effect is canceled if an array of lenses is considered. MPL filters are therefore competitive for scanning multi-slit systems.

Since the MPL is one-dimensional, it requires a lineshaped source as thin as $24 \mu \mathrm{m}$ and with an aspect ratio as high as 1:35 to keep a reasonable scan time. In general, the thinner the source, the better the energy filtering. Our calculations indicate that microfocal line sources with enough power for a competitive scan time are in principle feasible. We use a model that only takes heating of the anode into account, but more thorough studies, which include also heating of the cathode and the space charge effect, have concluded that microfocal sources are feasible for medical imaging [28]. Still, however, it is clear that less stringent demands on the focal spot size and/or the tube power would be advantageous.

In this study, we use mammography as an example. Advantages of the energy filter are, however, likely to be found also within other kinds of medical X-ray imaging, especially computed tomography where doses are high, and the broad energy spectrum leads to image artifacts such as beam hardening [6]. Small animal X-ray imaging is another field where dose reduction is needed since the required high resolution demands almost lethal dose levels [29]. In this case, the high resolution provided by an MPL filter is an additional advantage. Moreover, the tunability of the filter opens the possibility to conduct dual-energy imaging for contrast enhancement and detection of contrast agents at high sensitivity.

\section{Conclusions}

We conclude that MPL filtering is a promising technology for medical imaging, in particular for mammography, to reduce dose or to improve the signal-to-noise ratio. Our proposed ray-tracing and geometrical models agree well with measurements and can be used to predict the behavior of MPL filters.

The investigated epoxy MPL generates an $11 \mu \mathrm{m}$ wide one-dimensional image of the source at a peak energy of $20 \mathrm{keV}$, which is within one standard deviation of the expectations from geometry. The gain of flux is measured to
6.6, which is also in good agreement with the geometrical model. Ray tracing shows that deviations in the gain can be explained almost entirely by approximations in the model. Tunability is verified by altering the gap at the rear end of the lens to achieve peak energies of 17, 20, and $23 \mathrm{keV}$. The change in gap agrees within $2 \mu \mathrm{m}$ of model predictions.

Compared to a reference mammography system, the MPL filter reduces the dose by $13-25 \%$ for $3-7 \mathrm{~cm}$ breasts. This result depends on the absorption filter of the reference system but is virtually independent of the investigated anode materials, given the same anode in both systems. The resolution in the scanning direction is improved 2.5 times for a $5 \mathrm{~cm}$ breast, but the scan time is 3 times longer. If adapted to the same scan time as the reference system, the MPL filter is able to reduce the dose $16 \%$ at a 1.88 times better resolution. An MPL-based system does, however, require a thin line source, and the feasibility of such sources has to be investigated further.

\section{Acknowledgment}

We acknowledge the Swedish Research Council for funding parts of our work on multiprism lenses.

\section{References}

[1] J. W. Motz and M. Danos, "Image information content and patient exposure," Medical Physics, vol. 5, no. 1, pp. 8-22, 1978.

[2] R. J. Jennings, R. J. Eastgate, M. P. Siedband, and D. L. Ergun, “Optimal X-ray spectra for screen-film mammography," Medical Physics, vol. 8, no. 5, pp. 629-639, 1981.

[3] R. Fahrig and M. J. Yaffe, "Optimization of spectral shape in digital mammography: dependence on anode material, breast thickness, and lesion type," Medical Physics, vol. 21, no. 9, pp. 1473-1481, 1994.

[4] G. E. Pfahler, "A roentgen filter and a universal diaphragm and protecting screen," Transactions of the American Roentgen Ray Society, pp. 217-224, 1906.

[5] R. E. Johnston, D. Washburn, E. Pisano, et al., "Mammographic phantom studies with synchrotron radiation," Radiology, vol. 200, no. 3, pp. 659-663, 1996.

[6] F. A. Dilmanian, X. Y. Wu, E. C. Parsons, et al., "Single- and dual-energy CT with monochromatic synchrotron X-rays," 
Physics in Medicine and Biology, vol. 42, no. 2, pp. 371-387, 1997.

[7] R. Meuli, Y. Hwu, J. H. Je, and G. Margaritondo, "Synchrotron radiation in radiology: radiology techniques based on synchroton sources," European Radiology, vol. 14, no. 9, pp. 15501560, 2004.

[8] A. Krol, A. Ikhlef, J. C. Kieffer, et al., "Laser-based microfocused X-ray source for mammography: feasibility study," Medical Physics, vol. 24, no. 5, pp. 725-732, 1997.

[9] W. Knüpfer, "Feasibility study of a channeling radiation source for applications in the X-ray region," Nuclear Instruments and Methods in Physics Research B, vol. 87, no. 1-4, pp. 98-103, 1994.

[10] M. A. Piestrup, X. Wu, V. V. Kaplan, et al., "A design of mammography units using a quasimonochromatic X-ray source," Review of Scientific Instruments, vol. 72, no. 4, pp. 2159-2170, 2001.

[11] F. E. Carroll, "Tunable monochromatic X-rays: a new paradigm in medicine," American Journal of Roentgenology, vol. 179, no. 3, pp. 583-590, 2002.

[12] P. Baldelli, A. Taibi, A. Tuffanelli, M. C. Gilardoni, and M. Gambaccini, "A prototype of a quasi-monochromatic system for mammography applications," Physics in Medicine and Biology, vol. 50, no. 10, pp. 2225-2240, 2005.

[13] R. Lawaczeck, V. Arkadiev, F. Diekmann, and M. Krumrey, "Monochromatic X-rays in digital mammography," Investigative Radiology, vol. 40, no. 1, pp. 33-39, 2005.

[14] F. R. Sugiro, D. Li, and C. A. MacDonald, "Beam collimation with polycapillary X-ray optics for high contrast high resolution monochromatic imaging," Medical Physics, vol. 31, no. 12, pp. 3288-3297, 2004.

[15] B. Cederström, R. N. Cahn, M. Danielsson, M. Lundqvist, and D. R. Nygren, "Focusing hard X-rays with old LPs," Nature, vol. 404, no. 6781 , p. 951, 2000.

[16] B. Cederström, M. Lundqvist, and C. Ribbing, "Multi-prism X-ray lens," Applied Physics Letters, vol. 81, no. 8, pp. 13991401, 2002.

[17] D. A. Arms, E. M. Dufresne, R. Clarke, S. B. Dierker, N. R. Pereira, and D. Foster, "Refractive optics using lithium metal," Review of Scientific Instruments, vol. 73, no. 3, pp. 1492-1494, 2002.

[18] W. Jark, "A simple X-ray monochromator based on an alligator lens," X-Ray Spectrometry, vol. 33, no. 6, pp. 455-461, 2004.

[19] M. J. Berger, J. H. Hubbell, S. M. Seltzer, J. S. Coursey, and D. S. Zucker, "XCOM: Photon Cross Section Database," National Institute of Standards and Technology, Gaithersburg, Md, USA, 2005, http://physics.nist.gov/xcom.

[20] B. L. Henke, E. M. Gullikson, and J. C. Davis, "X-ray interactions: photoabsorption, scattering, transmission, and reflection at $E=50-30,000 \mathrm{eV}, \mathrm{Z}=1-92$," Atomic Data and Nuclear Data Tables, vol. 54, no. 2, pp. 181-342, 1993.

[21] M. Lundqvist, B. Cederström, V. Chmill, M. Danielsson, and B. Hasegawa, "Evaluation of a photon-counting X-ray imaging system," IEEE Transactions on Nuclear Science, vol. 48, no. 4, pp. 1530-1536, 2001.

[22] M. Åslund, B. Cederström, M. Lundqvist, and M. Danielsson, "Optimization of operating conditions in photon counting multi-slit mammography based on Si-strip detectors," in Medical Imaging 2006: Physics of Medical Imaging, vol. 6142 of Proceedings of SPIE, pp. 1-10, San Diego, Calif, USA, February 2006.

[23] M. Åslund, B. Cederström, M. Lundqvist, and M. Danielsson, "Scatter rejection in multislit digital mammography," Medical Physics, vol. 33, no. 4, pp. 933-940, 2006.
[24] M. Yoshimatsu and S. Kozaki, "High brilliance X-ray sources," in X-Ray Optics: Applications to Solids, H. T. Queisser, Ed., vol. 22 of Topics in Applied Physics, pp. 9-33, Springer, Berlin, Germany, 1977.

[25] J. M. Boone, "Glandular breast dose for monoenergetic and high-energy X-ray beams: Monte Carlo assessment," Radiology, vol. 213, no. 1, pp. 23-37, 1999.

[26] J. M. Boone, T. R. Fewell, and R. J. Jennings, "Molybdenum, rhodium, and tungsten anode spectral models using interpolating polynomials with application to mammography," Medical Physics, vol. 24, no. 12, pp. 1863-1874, 1997.

[27] A. K. Freund, "Mosaic crystal monochromators for synchrotron radiation instrumentation," Nuclear Instruments and Methods in Physics Research A, vol. 266, no. 1-3, pp. 461-466, 1988.

[28] X. Wu and H. Liu, "Clinical implementation of X-ray phase-contrast imaging: theoretical foundations and design considerations," Medical Physics, vol. 30, no. 8, pp. 2169-2179, 2003.

[29] E. L. Ritman, "Micro-computed tomography—current status and developments," Annual Review of Biomedical Engineering, vol. 6, no. 1, pp. 185-208, 2004. 